Original Article

\title{
Effect of complex training on carbon monoxide, cardiorespiratory function, and body mass among college students at the initial stage of stopping smoking
}

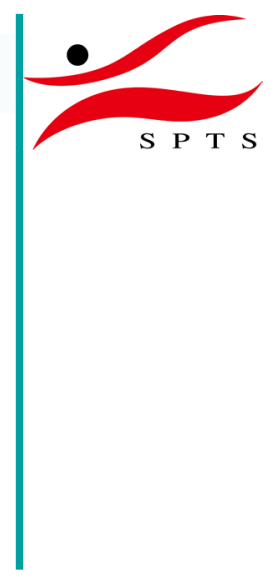

\author{
SEUnGSUK KIM, $\mathrm{PhD}^{1)}$ \\ 1) Department of Sports and Health Management, Mokwon University: Daejeon 302-729, \\ Republic of Korea
}

\begin{abstract}
Purpose] This study aimed to analyze the effects of complex training on carbon monoxide, cardiorespiratory function, and body mass among college students with the highest smoking rate among all age group. [Subjects and Methods] A total of 40 college students voluntarily participated in this study. All subjects smoked and were randomly divided into two groups: the experimental group $(\mathrm{N}=20)$ and the control group $(\mathrm{N}=20)$. The experimental group underwent complex training ( $30 \mathrm{~min}$ of training five times a week for 12 weeks) while the control group did not participate in such training. The complex training consisted of two parts: aerobic exercise (walking and running) and resistance exercise (weight training). [Results] Two-way ANOVA with repeated measures revealed significant interactions among CO, VO2max, HRmax, VEmax, body fat, and skeletal muscle mass, indicating that the changes were significantly different among groups. [Conclusion] A 12 week of complex physical exercise program would be an effective way to support a stop-smoking campaign as it quickly eliminates CO from the body and improves cardiorespiratory function and body condition.

Key words: Complex training, Cardiorespiratory function, Smoking
\end{abstract}

(This article was submitted Mar. 2, 2017, and was accepted May 9, 2017)

\section{INTRODUCTION}

In January 2015, the Korean government initiated a stop-smoking campaign as a means to enhance the overall health of the Korean people. The campaign increased cigarette cost and turned public places such as restaurants, bars, and coffee shops into non-smoking area. As a result of the stop-smoking campaign, the adult smoking rate decreased from $43.1 \%$ in 2014 to $39.9 \%$ in 2015 indicating the approach of a smoking rate below 30\%, which would be a first in the history of official smoking statistics ${ }^{1}$. However, the smoking rate has not decreased as markedly in college students. The smoking rate among college students in Korea is $23.2 \%$, well above the average reported by the Organization for Economic Cooperation and Development (OECD) of $20.7 \%{ }^{2}$. This discrepancy may be due to a change in college students once they reach the age at which they can buy cigarettes legally.

Cigarettes contain over 5,000 types of toxic or carcinogenic compounds including carbon monoxide (CO), nicotine, and tar. $\mathrm{CO}$ has a high capacity to chemically combine with hemoglobin $(\mathrm{Hb})$, thereby resulting in an elevation of the carboxyhemoglobin $(\mathrm{COHb})$ level thereby impairing the oxygen transportation activity of blood and resulting in polycythemia ${ }^{3)}$. In addition, $\mathrm{CO}$ decreases the oxygen level in blood resulting in damage to blood vessels and it has been associated with an increase in blood cholesterol, atherosclerosis, lung cancer, and colorectal cancer ${ }^{4,5}$. Typically, smokers have lower values for various bronchial resiliency indicators including forced vital capacity (FVC), forced volume for 1 second (FEV1), and 
Table 1. Changes in carbon monoxide, cardiorespiratory function, and body composition

\begin{tabular}{llcc}
\hline Items & Group & Pre & Post \\
\hline \multirow{2}{*}{ Carbon monoxide (ppm) } & Exercise & $18.6 \pm 3.4$ & $0.3 \pm 1.7^{*}$ \\
& Control & $17.3 \pm 2.6$ & $0.5 \pm 2.5$ \\
VO2max (ml/kg/min) & Exercise & $38.8 \pm 4.3$ & $43 \pm 2.7^{*}$ \\
& Control & $37.9 \pm 3.7$ & $38.3 \pm 3.9$ \\
HRmax (beat/min) & Exercise & $185.8 \pm 4.5$ & $195.8 \pm 2.6^{*}$ \\
& Control & $187.6 \pm 3.3$ & $189.2 \pm 3.1$ \\
VEmax (1/min) & Exercise & $64.3 \pm 1.6$ & $69.6 \pm 1.6^{*}$ \\
\multirow{2}{*}{ Body fat $(\%)$} & Control & $65.1 \pm 2.3$ & $66.1 \pm 2.6$ \\
\multirow{2}{*}{ Skeletal muscle (kg) } & Exercise & $26.8 \pm 2.6$ & $19.6 \pm 2.1^{*}$ \\
& Control & $25.2 \pm 1.6$ & $27.6 \pm 2.6$ \\
\hline
\end{tabular}

Values are expressed as mean \pm SD. Significantly different $(\mathrm{p}<0.05)$ between pre- and post-complex exercise training program.

forced expiratory flow (FEF) than those of non-smokers ${ }^{6}$. Moreover, the amount smoked is positively related to the body mass index (BMI) and abdominal obesity ${ }^{7,8}$. Therefore, smokers' abdominal circumference and abdominal obesity measures tend to be greater than those of non-smokers ${ }^{7,8}$. As college students have the highest smoking rate among all age groups ${ }^{1,2)}$, this study aimed to analyze the effect of complex training on exhalation $\mathrm{CO}$ level, cardiorespiratory function, and body mass in college students who were stopping smoking.

\section{SUBJECTS AND METHODS}

A total of 40 male college students voluntarily participated in this study. All subjects had been smoking for more than 1 year. Students diagnosed with a respiratory ailment by a doctor or who previously participated in a stop-smoking program were excluded from the study. The subjects were randomly divided into two groups: the experimental group $(\mathrm{N}=20 ; \mathrm{age}=20.2$ $\pm 0.3 \mathrm{yrs}$; height $=176.5 \pm 2.7 \mathrm{~cm}$; weight $=78.4 \pm 2.6 \mathrm{~kg})$ and the control group $(\mathrm{N}=20 ;$ age $=20.9 \pm 0.3 \mathrm{yrs} ;$ height $=175.7 \pm$ $1.7 \mathrm{~cm}$; weight $=79.2 \pm 2.0 \mathrm{~kg}$ ). All subjects had a 12 week of nonsmoking period from the beginning of the experiment. The experimental group underwent complex training while the control group did not participate in such training. All subjects were informed of the content and purpose of the experiment and all provided written informed consent (Mokwon University) before participating in the study.

Subjects' exhalation CO level, maximum oxygen uptake (VO2max), maximum heart rate (HRmax), maximum ventilation (VEmax), body fat content (\%), and skeletal muscle mass were measured at the start and end of the study. Exhalation CO level was measured to the nearest parts per million $(\mathrm{ppm})$ as previously reported ${ }^{9)}$ by using a $\mathrm{CO}$ measuring instrument (Micro Co., Rochester, Kent, England). For reference, CO levels were categorized into five stages: stage 1 (non-smoker, 0-6 ppm), stage 2 (risky, 7-10 ppm), stage 3 (smoker, 11-15 ppm), stage 4 (habitual smoker, 16-25 ppm), and stage 5 (addictive smoker, 26-35 ppm) as previously described ${ }^{9)}$.

Exercise tolerance was estimated by using the Bruce protocol. Briefly, the protocol begins with an initial treadmill speed of 1.7 miles per hour ( $\mathrm{mph}$ ), which is then increased by $0.8 \mathrm{mph}$ every $3 \mathrm{~min}$; in addition, the initial gradient of $10 \%$ is increased by $2 \%$ every 3 min. Subject's VO2max, HRmax, and VEmax were estimated by using a breath-by-breath respiration analysis gas analyzer assembly (Quark b2; Cosmed, Rome, Italy), which was operated in peak mode.

Body composition determined from body fat content $(\%)$ and skeletal muscle mass $(\mathrm{kg})$ values was estimated by using bioelectrical impedance analysis equipment (InBody J20, Bio-Space, Seoul, Korea).

The complex training program included participation in physical exercise for $30 \mathrm{~min}$ five times a week for 12 weeks at the fitness center of D university. The complex training program consisted of two parts: aerobic exercise (walking and running) and resistance exercise (weight training). Aerobic exercise was performed until the subject attained $50-70 \%$ of their HRmax. To accurately assess exercise intensity, subject heart rate was monitored during exercise with a portable recordable HR monitor (Polar Electro, Technogym, Finland). Weight training was performed until $70 \%$ of a subject's one repetition maximum (1RM) was attained, with the 1RM values calculated by using Brzycki's equation ${ }^{10)}$. Table 1 summarizes the complex training program.

All experiments were reviewed and approved by the Institutional Review Board of Daegu University. The data obtained were processed by using SPSS 20.0 version (IBM, Armonk, NY, USA). Mean and standard deviation values of the two groups were calculated and two-way ANOVA with repeated measures was conducted to examine differences between the groups and between measurement times. Values of $\mathrm{p}<0.05$ were considered statistically significant. 


\section{RESULTS}

The two-way ANOVA with repeated measures showed significant interactions $(\mathrm{p}<0.05)$ among CO, VO2max, HRmax, VEmax, body fat content, skeletal muscle mass, and waist: hip ratio; thus, indicating that the changes were significantly different between the groups.

\section{DISCUSSION}

The Korean government has been attempting to decrease the adult smoking rate below $29 \%$ by implementing new policies, such as raising the cost of cigarettes and placing graphic health warnings on cigarette packages ${ }^{1)}$. Despite these policies, the smoking rate of college students, in particular, has not been decreasing as much as other groups ${ }^{1,2)}$. There are many health concerns related to smoking. One, in particular, is $\mathrm{CO}$, which has a high affinity to chemically combine with $\mathrm{Hb}$, thereby impairing the oxygen transportation capacity of smokers' blood, as well as affecting cardiorespiratory function and blood pressure $\left.{ }^{3,11}, 12\right)$. Smokers tend to have approximately $10 \%$ lower oxygen transportation capacity than non-smokers ${ }^{13)}$. In addition, the associated higher level of carboxyhemoglobin increases the possibility developing coronary artery disease, cerebral infarction, chronic obstructive lung disease, and lung cancer $\left.{ }^{14}, 15\right)$. On that basis, this study aimed to analyze the effects of participation in complex training on $\mathrm{CO}$, cardiorespiratory function, and body mass among college students who were at the initial stage of stopping smoking. The results of this study show that exhalation CO levels decreased in both the experiment and control groups. Notably, the exhalation $\mathrm{CO}$ level of the experiment group significantly decreased from stage 4 (habitual smoker, CO level 16-25 ppm) to stage 1 (non-smoker, CO level 0-6 ppm). The control group also had a decreased exhalation $\mathrm{CO}$ level; however, the experimental group had a greater $\mathrm{CO}$ decrease. Complex training assisted in achieving this result by increasing blood circulation, transport of oxygen, heartbeat, respiration volume, pulmonary circulation, and perspiration. In addition, the experimental group showed significant increases in VO2max, HRmax, and VEmax indicating multiple effects of the complex training program. In contrast, the control group did not show such increases. The results of this study are consistent with those in previous studies. Rode and Shephard ${ }^{16)}$ examined the effect of smoking and abstinence from cigarette on the oxygen cost associated with hyperventilation. The hyperventilation oxygen cost was measured twice, immediately following smoking and after one day of abstinence from cigarettes ${ }^{16)}$. After abstinence, the hyperventilation oxygen cost was $13-79 \%$ lower than that before abstinence ${ }^{16)}$. Byun ${ }^{17)}$ reported that 8 weeks of a combined exercise training program significantly improved VO2max, body composition, and, in particular, body fat content among current smokers. Nam and Kang ${ }^{18)}$ analyzed changes in CO level in two groups over a 12 week period. They ${ }^{18)}$ found that those abstaining from cigarettes and exercising had a greater decrease of $\mathrm{CO}$ level than those abstaining from cigarettes but not exercising.

The results of the present study also showed changes in body fat (\%), waist: hip ratio, and skeletal muscle mass (kg). The body fat content $(\%)$ decreased and skeletal muscle mass $(\mathrm{kg})$ increased only in the experiment group. These results are consistent with those in previous studies ${ }^{19,20)}$. Those studies ${ }^{19,20)}$ suggested that complex physical exercise increases basal metabolism by developing skeletal muscles and such exercise also reduces obesity, diabetes, and many other chronic diseases. In addition, development of skeletal muscles reflects associated increases in mitochondria, oxidative enzymes, muscle capillaries, and muscle blood flow rate ${ }^{19,20)}$. In conclusion, a complex physical exercise program would be an effective way to support a stop-smoking program as it can quickly eliminate $\mathrm{CO}$ from the body and improve cardiorespiratory functions and body mass. Therefore, this study provided practical data establishing the effective exercise program for people who are stopping smoking and want to improve their health (e.g., cardiorespiratory function, and body mass). However, this study only focused on male college students. Thus, future study needs to include diverse personal characteristics, such as age and gender.

\section{REFERENCES}

1) Korea Ministry of Health and Welfare: Korea Health Statistics. Seoul: Korea Ministry of Health and Welfare, 2016.

2) Lee HS, Song MR: The effects of a quit smoking program using the web and short message service on exhaled carbon monoxide, self-efficacy and depression according to nicotine dependency level in undergraduates students. J Korean Biol Nurs Sci, 2014, 16: 173-181. [CrossRef]

3) Chung KI, Jang MJ, Nho HS: The effects of cigarette smoking and exercise habit to cardiovascular risk factor in 40's men. Kor J Sport Sci, 2009, 18: 969-978.

4) Preston SH, Glei DA, Wilmoth JR: A new method for estimating smoking-attributable mortality in high-income countries. Int J Epidemiol, 2010 , 39: 430-438. [Medline] [CrossRef]

5) Vogelaar I, van Ballegooijen M, Schrag D, et al.: How much can current interventions reduce colorectal cancer mortality in the U.S.? Mortality projections for scenarios of risk-factor modification, screening, and treatment. Cancer, 2006, 107: 1624-1633. [Medline] [CrossRef]

6) Cha KS: A comparison of pulmonary function aerobic power blood pressure blood hemoglobin in smokers and non-smokers. Kor J Phys Educ, 2001, 40: $845-857$.

7) Molarius A, Seidell JC, Kuulasmaa K, et al.: Smoking and relative body weight: an international perspective from the WHO MONICA Project. J Epidemiol Community Health, 1997, 51: 252-260. [Medline] [CrossRef]

8) Seidell JC, Cigolini M, Deslypere JP, et al.: Body fat distribution in relation to physical activity and smoking habits in 38-year-old European men. The European Fat Distribution Study. Am J Epidemiol, 1991, 133: 257-265. [Medline] [CrossRef] 
9) Kwon OY, Song JH, Park KS: Effects of auricular acupressure therapy on decreasing smoking rates, desire for smoking, and exhaled carbon monoxide in male college students. Korean J Rehabil Nurs, 2015, 18: 88-97. [CrossRef]

10) Brzycki M: Strength testing: predicting a one-rep max from reps-to-fatigue. J Phys Educ Recreat Dance, 1993, 64: 88-90. [CrossRef]

11) Nam SN, Kang SH: Research on changes in in-body expiration carbon monoxide and nicotine according to physical exercise during non-smoking sessions. Exerc Sci, 2013, 22: 233-238. [CrossRef]

12) Chang IH, Lee SK: Cardiorespiratory functions of smoking athletes in maximal exercise. Kor J Sport Sci, 2000, 9: 421-432.

13) Fox EL: Sports physiology. PA: Saunders College Pub, 1984.

14) Hart CL, Smith GD, Hole DJ, et al.: Carboxyhaemoglobin concentration, smoking habit, and mortality in 25 years in the Renfrew/Paisley prospective cohort study. Heart, 2006, 92: 321-324. [Medline] [CrossRef]

15) Choi DW, Yoon JH, Jung SH, et al.: Effects of 16 weeks treadmill exercise on endothelial function and plasma ferritin levels in adolescent smokers. J Kor Soc Living Environ Sys, 2013, 20: 681-688.

16) Rode A, Shephard RJ: The influence of cigarette smoking upon the oxygen cost of breathing in near-maximal exercise. Med Sci Sports, 1971, 3: 51-55. [Medline]

17) Byun JC: Effects of aerobic and anaerobic combined exercise training on body compositions, cardiovascular variables, blood lipids and acute inflammatory markers in male smoker and non-smokers during 8 weeks. Kor J Sport Sci, 2008, 19: 51-59. [CrossRef]

18) Nam YS, Kim GT: Effects of circuit weight training on muscular strength and cardiopulmonary functions in smoking cigarettes. Kor J Sport Sci, 2004, 13: $683-690$.

19) Williams MA, Haskell WL, Ades PA, et al. American Heart Association Council on Clinical Cardiology American Heart Association Council on Nutrition, Physical Activity, and Metabolism: Resistance exercise in individuals with and without cardiovascular disease: 2007 update: a scientific statement from the American Heart Association Council on Clinical Cardiology and Council on Nutrition, Physical Activity, and Metabolism. Circulation, 2007,116 : 572-584. [Medline] [CrossRef]

20) Nam SN, Kang SH: The effects of physical exercise during smoking inhibition session of smokers, on in-body nicotine and expiration carbon monoxide. Exerc Sci, 2013, 22: 233-238. 